\title{
A Co-Creation Platform for Creative Engagement of End-Users in a Connected Object Space
}

\author{
Johan Criel, Geert Vanderhulst, Fahim Kawsar, Lieven Trappeniers \\ Bell Laboratories, Alcatel-Lucent, Belgium \\ \{johan.criel, geert.vanderhulst, fahim.kawsar, lieven.trappeniers\}@alcatel-lucent.com
}

\begin{abstract}
The proliferation of open source hardware platforms and consequent connected objects has created novel design opportunities for end-users to realize their personalized "lifehacks" in an interactive environment. However, this lifehack culture is yet to become a mainstream practice due to the complexity of the creation process, application provisioning and the lack of appropriate distribution channels. In this paper, we address the first two issues and present a system platform that empowers ordinary individuals to create and share their personalized sensor driven applications in a highly abstract manner towards a co-creation ecosystem. Essentially, our platform enables users to i) attach personal concepts (abstraction) to sensor data, ii) create personalized visualization of sensor data and iii) share these concepts and visualizations with others to facilitate in-situ search and a co-creation experience. We present the design space and technical description of the corresponding system platform. A feasibility study suggests that the proposed approach is simple, comprehensive and has the potential for facilitating creativity in the connected object space.
\end{abstract}

\section{ACM Classification Keywords}

D.2.11 Software Engineering: Software Architectures

\section{Author Keywords}

Connected Objects, End-User Development, System Infrastructure, Social Computing

\section{General Terms \\ Design, Experimentation, Human Factors}

\section{INTRODUCTION}

We are observing two significant metamorphosis of our computation driven experience - firstly, a plethora of open source hardware - i.e. sensors and actuators - resulting in more and more connected objects embodied with intelligence and secondly, a paradigm shift of our personal interaction instigated by social computing platforms. The former led by

Permission to make digital or hard copies of all or part of this work for personal or classroom use is granted without fee provided that copies are not made or distributed for profit or commercial advantage and that copies bear this notice and the full citation on the first page. To copy otherwise, or republish, to post on servers or to redistribute to lists, requires prior specific permission and/or a fee.

NOMe-IoT'11, September 18, 2011, Beijing, China.

Copyright 2011 ACM 978-1-4503-0930-1/11/09...\$10.00. a number of industrial effort (e.g. Arduino, Phidget, ...) has created opportunities for ordinary individuals to build imaginative new forms of interaction and sensor-driven long tail ${ }^{1}$ applications for everyday objects around us. While end-user development for physical computing has been an active area in the academic community for quite a while $[2,3,5,7,8,10]$, these platforms really pushed the innovation ability to end-users' hands and afforded them to develop sensor-driven applications. In parallel, data aggregator services (e.g. Pachube ${ }^{2}$ ) and their respective open APIs have provided the foundation for opportunistic data sharing.

On the other hand, the phenomena of social computing undoubtedly transformed our lifestyle. Online social services like Facebook, Twitter, Flickr, etc. have changed the way we share our lives with our friends and family. Projecting into the future, we can imagine that soon there will be a strong coupling between connected objects and social networks. For instance, a "Green" person might instrument her everyday objects to generate her accumulated personal carbon footprint and share it in the social network to compete with her friends. An "away-from-home" individual might engage in a more empathic social interaction through object instrumentation. Connected objects will give a new meaning to our social life and will play an active role in the process of how we learn, think, and behave as social human beings.

Yet, while there are unprecedented opportunities to instrument and share personalized physical objects, we observe that aforementioned services are still popular to only a certain category of users - the so-called "Technology Hacktivists". To attach an Arduino to a bike for monitoring personal carbon footprint and sharing it with Pachube, an individual needs to spend a serious amount of effort in learning the Arduino and Pachube programming, and moving to another platform will require further learning effort. In addition, the creation mechanism such as assigning meaning to a sensor value and fusing sensor feeds to create an input mashup, is seriously difficult. At the same time, the eventual sensor values do not make any sense unless they are mapped to a suitable visualization. Furthermore, at this point there is no common space for individuals where end-users' creation efforts (concept, instrumentation, visualization, etc.) can be shared with others thus limiting a co-creation ecosystem.

To address these challenges, we present a user centric soft-

\footnotetext{
${ }^{1}$ http://www.wired.com/wired/archive/12.10/tail.html

${ }^{2}$ http: / / www . pachube.com
} 
ware platform that radically simplifies physical object instrumentation by offering end-users with the ability to attach high-level concepts to sensors isolating low-level sensor programming, to visualize concepts in a personalized way, and finally to share both their concepts and visualizations socially. In this note, we concisely describe the design principles and implementation of this platform and present a feasibility study as a verification of the effectiveness of our platform in offering a co-creation experience to end-users.

\section{DESIGN PRINCIPLES \& WORKFLOW}

For creating a co-creation platform that can support endusers' social creativity in the connected object space, we observe that there are three main challenges the software platform must address: i) enabling an individual to attach personal concepts to sensors, ii) incorporating sharing and searching mechanisms and finally iii) an easy to use creation environment to design visualizations. In this section, we discuss how these principles are addressed in our system. Users can add their personalized meaning to sensors by defining high-level concepts that act as an abstraction for these sensors. Examples of concepts include statements that can be measured such as "person is having breakfast", "person is cycling", "curtain is open or closed" and so on. Users can share these concepts so that they can be reused by others in the same or even another domain. Each concept is semantically annotated with its context of use (e.g. the smart objects related to it, tags, ...) which enables rich searching capabilities. A concept can be implemented in different ways based on the type of sensors at hand. For example, a "curtain open or closed" concept could be implemented using an IR distance Phidget sensor or a magnetic Arduino sensor, both having their respective implementation and installation guide.

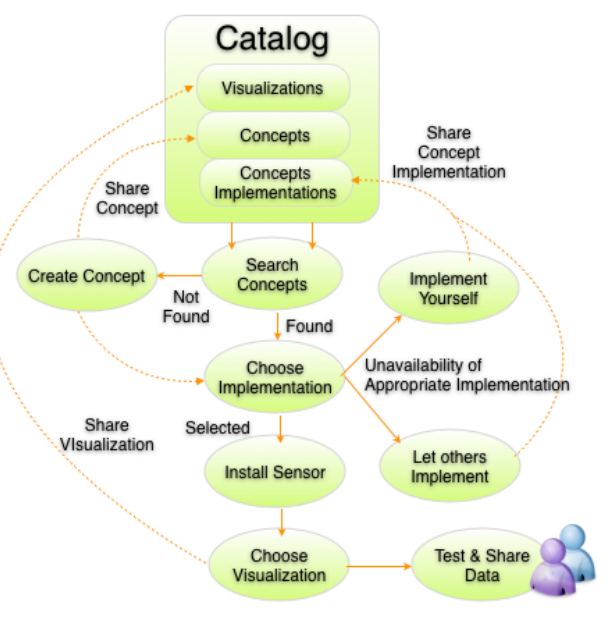

Figure 1. Workflow of co-creation ecosystem.

We distinguish four different levels of sharing: i) concept level, ii) concept implementation level, iii) visualization level, iv) data level. New concepts are shared during the creation process and become available for others' use, i.e. to install them in their environment or to attach a new implementation to a concept. These implementations can be shared by writ- ing an installation manual and defining configuration templates. Similarly, the actual data produced and transformed by a concept implementation can be shared explicitly, either as raw data or as a visualization of this data. This sharing functionality gives rise to a community of users that get inspired by each other's concepts. For instance, users can get notified when new concepts (and their evolving implementations) become available that can be realized using the sensors a user already has.

Users can create their own interactive visualizations related to concepts or use existing ones shared by others. The visualized data can be based on one or more concepts and can vary from traditional real-time adapted charts to custom made interactive compositions. Currently, our platform offers a simple visual editor where end-users can interact with underlying sensors and transformers, abstracted by concepts, to create visualizations. In addition, an individual can share this visualization, as well as the underlying implementation.

It is also possible to link visualization templates to a concept in the catalog. As such, users are able choose an appropriate visualization created by others while installing a concept and to map corresponding visulaizations using sliders and text buttons, threshold values are set that transform the sensor data. Visualizations can be shared together with the data, which means that the person gets access not only to the data but also to the visualization of his friends. As for concepts it is possible to directly integrate these visualizations in an application or adapt it to your domain and personal preferences/creativity.

Figure 1 depicts the flow of the creation process of a new concept and the usage of existing concepts. The co-creation ecosystem relies on a central catalog where all concepts and their implementations are stored. The catalog allows users to search for concepts based on their preferred mental model. Users can search for concepts using keywords and then select an appropriate implementation that can be installed using sensors that they possess. Alternatively, a user can ask the system which concepts others have created with one or combination of sensors she currently owns as a source of inspiration. When a concept is not available in the catalog a user can define and implement it herself or can delegate to experts in the community.

After selecting a concept and (one of) its implementation(s), the user is guided through an installation step to make the concept available in her physical environment. This is followed by a configuration phase where the user can specify and finetune how the concept's data should be visualized and whether the user wants to explicitly share this data with others. The testing phase completes the installation after the user verified and confirmed that everything is working based on the visualization that is presented (i.e. are all sensors properly connected and providing the expected information?).

\section{IMPLEMENTATION}




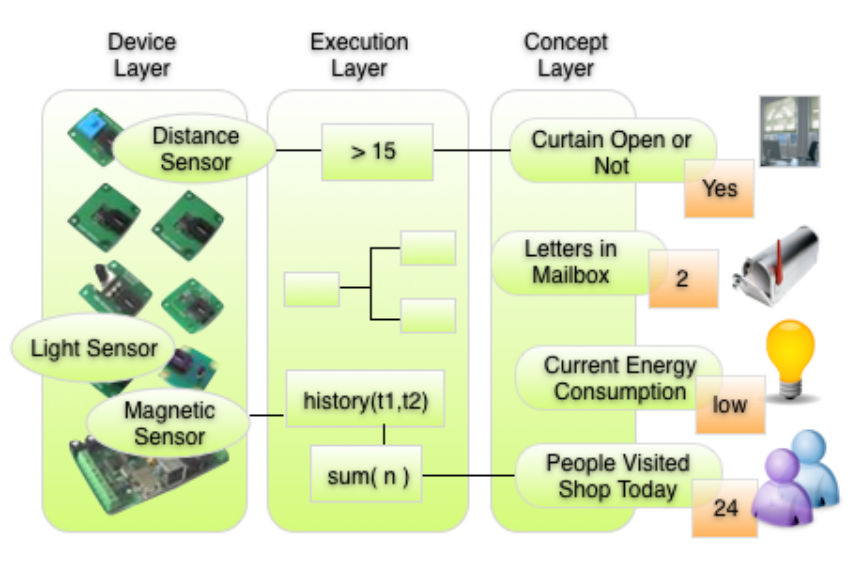

Figure 2. Bird's Eye View on Platform Architecture

Figure 2 shows a bird-eye architectural view of our cocreation platform which consists of three major layers that are interconnected through open REST interfaces. The device layer integrates sensor devices that produce raw data. This data is forwarded to a distributed execution layer where it passes through one or more components that transform and aggregate the data into situation-specific information. This processed information feeds a concept layer defined by a user.

The end-user created concepts are defined in an ontology that evolves over time. These high-level concepts can be annotated with related objects, defined in the WordNet ${ }^{3}$ lexicon in order to avoid ambiguity and to improve the accuracy of search results by employing linguistic relations. For the concept implementations end-users can make use of high level building blocks that provide easy ways for transforming sensor data to the right level of abstraction, counting, filtering, etc. For creating these concepts end-users are offered a simple mash-up creation environment as shown in Figure 3(a). End users can connect multiple data sources, compose these data sources, and filter them with personalized behaviour by attaching rules. The creation environment is built with $\mathrm{JGraph}^{4}$ library that offers highly flexible drag and drop interaction features for creating mashups. Dedicated, more complex transformation blocks can be added by more experienced users that have some knowledge on scripting. When a concept implementation is shared, the platform offers support to the creator to extract a configuration template that includes other high level concepts or sensors that are needed as inputs, a guide on how these physical sensors should be installed and the possible limitations of the implementation. When an end-user installs a concept, the high level building blocks associated with the end-user chosen concept implementation are translated in machine understandable component mash-ups. These mash-ups are then mapped, deployed and provisioned on the distributed processing nodes based on the network, sensor and actuator topology.

The co-creation platform also offers a small set of APIs aimed

\footnotetext{
${ }^{3}$ http://wordnet.princeton . edu/

${ }^{4}$ http://www.jgraph.com/
}

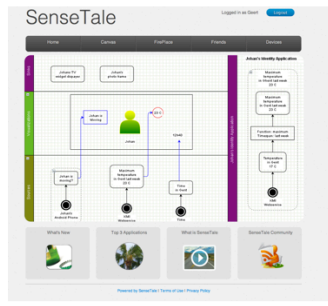

(a)

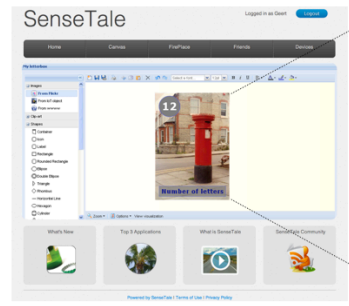

(b)

\section{Figure 3. (a) Creation Environemnt and (b) Visualization Editor}

towards seasoned end-users to connect their sensor devices to the platform. These APIs allows them to create a wrapper of their native sensor devices, so that the devices' data can be published to our co-creation platform and henceforth can be used in the creation environment as shown in Figure 3(a).

End-users have a visual interface that allows them to create their own visualizations as illustrated in Figure 3(b). To personalize a visualization, users can insert pictures such as a letterbox, which represent their physical environment. Moreover, they can drag different kind of shapes on the visualization canvas and link them to installed concepts. The real-time data produced by these concepts is then presented inside the shape. Note that this data originates from the execution layer where it is transformed and pushed to the visualization using the AJAX-Comet paradigm. For instance, in the figure the number of letters currently in the mailbox in depicted in a circle on the mailbox image. Moreover, users can define visual effects that are triggered by simple rules. For example, users can define conditional statements such as if my mailbox is full (more than 10 mails), show a red flag on my mailbox. The combination of real-time data and visual effects enables users to quickly interpret the current context of the environment though personalized visualizations. Similar to concepts, user defined visualizations can be shared and reused by others.

\section{EVALUATION: A PILOT STUDY}

In the earlier sections we have concisely described the design and implementation of the co-creation platform. We see two alternatives for evaluating this platform: i) system benchmarking and ii) end-user evaluation. Considering the fact that this note primarily discusses the user aspect, we present in this section a small-scale pilot user study evaluating the usability of this platform and its underlying mechanisms to support end-users in creating and sharing connected object concepts. For study design, we have followed the methodology suggested and used in $[4,8]$.

\section{Study Methodology}

We recruited 12 ordinary individuals ( 7 males 5 females, age range between 26 and 39) with moderate experiences in using mobile phones and computers. We provided these participants with a sensor kit containing six different sensors (distance, magnetic, temperature, light, pressure and humidity). The sensors were connected to our co-creation platform through an InterfaceKit board. In addition to the 


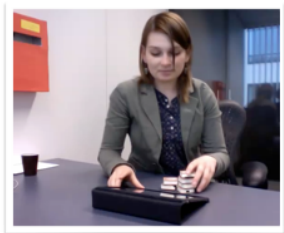

(a)

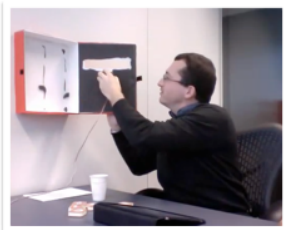

(b)

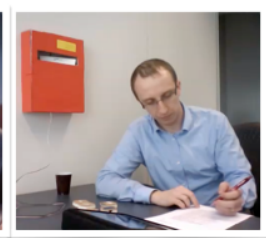

(c)
Figure 4. Participants (a) working on the platform (b) installing sensors on the letter box and (c) answering questionnaires.

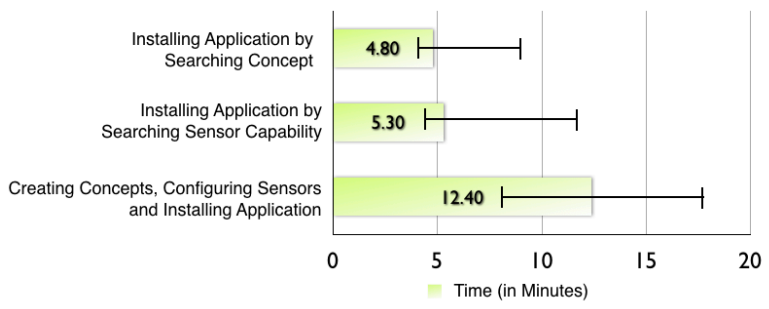

Figure 5. Completion time for different activities.

sensor kit, we provided the participants with an Apple iPad running the web interface of the co-creation platform. Each study session took about 75 mins and was divided into three phases. In phase one we introduced the concept, co-creation platform, sensor kit and presented a tutorial. In the second phase, participants were given three activities of which the order was randomized:

- Activity 1: Searching with keywords for a concept and corresponding application in relation to a letter box that notifies when a new letter arrives, and once found, installing it using the sensor kit and co-creation platform.

- Activity 2: Identifying the capabilities of a magnetic sensor through searching and then installing an application that can detect whether curtain is open or not.

- Activity 3: Creating a concept "Thirsty Plant" using a temperature and a humidity sensor and creating a simple visualization of the concept from available templates.

Finally, in phase three, we had a questionnaire and in-depth follow-up semi-structured interview session. The questionnaire contained 16 statements structured with a five-point Likert scale to indicate their level of agreement or disagreement. The first ten statements were designed following the System Usability Scale (SUS) [1] and the remaining six questions regarding the complexities of different tasks involved in the activity We also asked about task demands following NASA TLX [6]. After the questionnaire, we interviewed users to gain further insights on their assessments. Each session was video taped for later analysis. Figure 4 shows some snapshots from the experiment sessions. As an incentive, each participant received a sensor kit.

\section{Study Results}

During the experimentation sessions, we measured the completion time for each of the activities. Figure 5 shows the

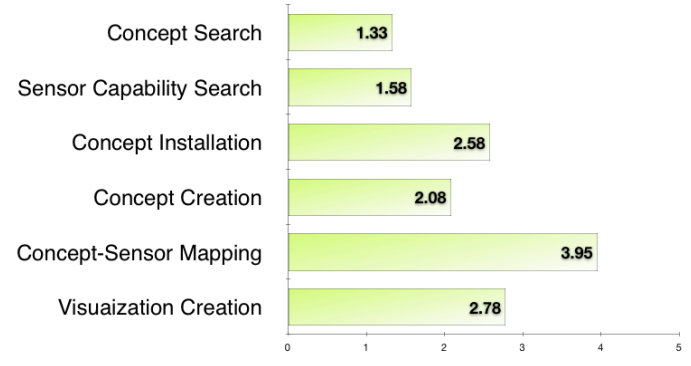

Complexity Scale [1: Very Easy 5: Very High]

Figure 6. Perceived complexity of different tasks.

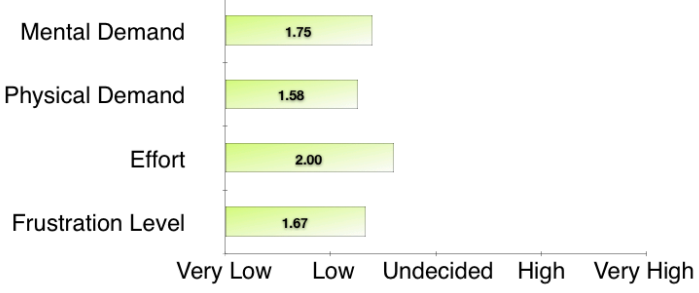

Figure 7. Average NASA Task Load Index.

average completion time. As expected, activity 1 (avg. 4.8 $\mathrm{min}$ ) and activity 2 (avg. $5.3 \mathrm{~min}$ ) required less time than activity 3 (avg. $12.40 \mathrm{~min}$ ). The slight difference between activity 1 and 2 could be due to the fact that end-users had to understand the role of a sensor before proceeding to the installation (activity 2) whereas for activity 1 they could just follow the installation instructions. The creation phase took longer time perhaps due to the facts that participants had to understand the normalized value of sensors and had to use proper transformation wiring from the platform's creation environment.

During the interview session, we asked the participants to mark the perceived complexity level as depicted in Figure 6. Participants generally found the tasks pretty simple. The only task that appeared complicated was the "concept to sensor mapping task" which in our co-creation platform is accomplished using a visual editor: by interacting with sliders and text buttons, threshold values are set that transform the sensor data. Currently, this creation environment is still limited and participants suggested us for further exploration, as they found the interaction experience rather complicated.We have also asked the participants about physical and mental demands, frustration level and needed effort for all three tasks following the NASA-TLX . Figure 7 summarizes the participants' responses. The overall response yielded fairly low task load demand. Subjective questions later revealed that the effort scored slightly higher perhaps due to the fact that participants had to familiarize with the whole system and the sensor kit.

The composite SUS score was 76.92 out of 100 (Standard Deviation: 8.84, Max: 90.0, Min: 62.5) regarding the overall usability of the co-creation platform and the process. We 


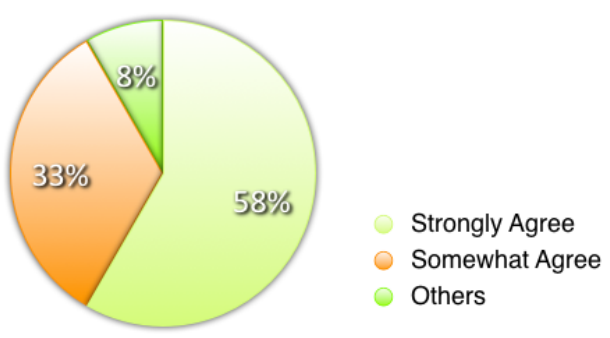

Figure 8. Individual preference towards acceptance of the co-creation platform

consider these values are quite promising. Moreover, the individual frequency (Figure. 8) of the acceptance statement in SUS: "I would like to have this system if it were available" [Strongly Agree: N=7 (58\%), Somewhat Agree: $\mathrm{N}=4(33 \%)$ ] suggests a positive response regarding the acceptance of the approach.

\section{IMPLICATIONS AND CONCLUSIONS}

The semi-structured interviews with the participants were coded in the analysis software MAXQDA, analyzed and discussed by the researchers. The analysis of these interviews combined with the quantitative findings revealed several interesting aspects regarding their understanding and qualitative assessments of the co-creation platform and the entire user experience. Participants repeatedly suggested that the main advantage of our platform was the sharing and searching feature as it allowed them to explore the possible concept space. One participant mentioned "I am not that creative, so probably I won't be able to come up with my own idea all the time unless I have a specific need, so this searching is useful.". Similar comments were received from other participants too. Extrapolating on their comments we suggest that it is absolutely necessary to support a community-driven development approach for the proliferation of such co-creation platform. Our suggestion can be justified further by looking at the popular DIY hacker site Instructables ${ }^{5}$ where social sharing of life-style hacks are extensively used. Participants also appreciated the high level concept definition aspect and majority of the participants preferred searching for concepts using keywords over searching for concepts based on the capabilities of a sensor. One interesting comment was "This is like a recipe for cooking: most of the time I want to eat food of a particular type, not the food that I can make with the ingredients I have in my house although this might be useful too.". Considering this feedback, we argue that introducing high-level concepts benefits the end-users and encourages them to use a platform like ours more frequently. One final aspect that came out during the interviews is the playful nature of the entire system. Most of the participants suggested that such platform would mostly be used by enthusiasts for personalised objects or fun like Lego Mindstroms ${ }^{6}$. This is a critical point as it exposes the fact that connected objects are still considered very lightly. Even though participants liked the concepts they created during the experiment, they men-

\footnotetext{
5http://www.instructables.com

${ }^{6}$ http: //mindstorms. lego.com
}

tioned that the value proposition is still not strong enough for mass penetration. This again opens up the argument that we are missing a set of concept-based applications that could significantly elevate the user centric values for connected objects. Recently Kortuem and Kawsar [9] argued for a market based user innovation for diffusion of connected objects following the success of user-driven innovation in the smart phone platform. We agree with their argument, and we hope to extend our social sharing aspect into more concrete distribution channels for the connected object space.

\section{REFERENCES}

1. J. Brooke. SUS: A quick and dirty usability scale, pages 189-194. Usability Evaluation in Industry. Taylor and Francis, London, 1996.

2. A. K. Dey, R. Hamid, C. Beckmann, I. Li, and D. Hsu. a cappella: Programming by demonstration of context-aware applications. In ACM CHI 2004, 2004.

3. A. K. Dey, T. Shon, S. Streng, and J. Kodama. Supporting end user programming of context-aware applications. In Pervasive 2006, 2006.

4. W. K. Edwards, V. Bellotti, A. K. Dey, and M. W. Newman. The challenges of user-centered design and evaluation for infrastructure. In CHI, pages 297-304, 2003.

5. K. Gajos, H. Fox, and H. Shrobe. End user empowerment in human centered pervasive computing. In International Conference on Pervasive Computing, 2002.

6. S. G. Hart and L. E. Staveland. Development of NASA-TLX (Task Load Index): Results of empirical and theoretical research, volume 1, pages 139-183. North-Holland, 1988.

7. J. Humble, A. Crabtree, T. Hemmings, B. K. Karl-Petter Åkesson, T. Rodden, and P. Hansson. Playing with your bits': user composition of ubiquitous domestic environments. In Ubicomp 2003, 2003.

8. F. Kawsar, T. Nakajima, and K. Fujinami. Deploy spontaneously: supporting end-users in building and enhancing a smart home. In Proceedings of the 10th international conference on Ubiquitous computing, UbiComp '08, pages 282-291, 2008.

9. G. Kortuem and F. Kawsar. Market-based user innovation in the internet of things. In IOT, 2010.

10. T. Yonezawa, H. Sakakibara, K. Koizumi, S. Miyajima, J. Nakazawa, K. Takashio, and H. Tokuda. upackage - a package to enable do-it-yourself style ubiquitous services with daily objects. In $U C S$, pages $240-257$, 2007. 